\title{
High-Gain SIR Dual-Band Antenna Based on CSRR-Enhanced SIW for 2.4/5.2/5.8 GHz WLAN
}

\author{
Caixia Feng $\mathbb{D}^{1,2,3}$ Yongqiang Kang, ${ }^{1,2,3}$ Lijuan Dong, ${ }^{1,2,3}$ and Lihong Wang ${ }^{3}$ \\ ${ }^{1}$ Institute of Solid State Physics, Shanxi Datong University, Datong, Shanxi 037009, China \\ ${ }^{2}$ Shanxi Provincial Key Laboratory of Electromagnetic Functional Materials for Microstructure, Shanxi Datong University, \\ Datong, Shanxi 037009, China \\ ${ }^{3}$ College of Physics and Electronics, Shanxi Datong University, Datong, Shanxi 037009, China
}

Correspondence should be addressed to Caixia Feng; fcxdtdx@163.com

Received 7 April 2020; Revised 4 July 2020; Accepted 13 July 2020; Published 29 July 2020

Academic Editor: Ding-Bing Lin

Copyright (C) 2020 Caixia Feng et al. This is an open access article distributed under the Creative Commons Attribution License, which permits unrestricted use, distribution, and reproduction in any medium, provided the original work is properly cited.

\begin{abstract}
This paper presents a dual-band step impedance resonator (SIR) antenna based on metamaterial-inspired periodic structure of coupled complementary split-ring resonators substrate-integrated waveguide (CSRR-SIW). The antenna supports wireless local area networks (WLAN) bands at 2.4/5.2/5.8 GHz. The CSRRs and two branches of the SIR element are etched on the top and bottom metal surfaces of the substrate. The SIR element produces a fundamental frequency $f_{1}$ at $2.4 \mathrm{GHz}$ and a second harmonic frequency $f_{\mathrm{s} 2}$ at $5.7 \mathrm{GHz}$. Meanwhile, the CSRRs produces a resonant frequency at high-frequency band around $5.2 \mathrm{GHz}$, which can be combined with the second harmonic frequency $f_{\mathrm{s} 2}$ at $5.7 \mathrm{GHz}$. The high-frequency bandwidth can then be broadened. The simulated and measured results show that the dual operation bands with bandwidths of $16 \%$ from $2.25 \mathrm{GHz}$ to $2.64 \mathrm{GHz}$ and $18.2 \%$ from $5 \mathrm{GHz}$ to $6 \mathrm{GHz}$ for $|S 11|<-10 \mathrm{~dB}$ are achieved. Meanwhile, the proposed antenna has peak gains ranging from $6.5 \mathrm{dBi}$ to $7 \mathrm{dBi}$ and from $7 \mathrm{dBi}$ to $7.7 \mathrm{dBi}$ in the lower and upper bands, respectively. Compared with many previously reported dual-band antenna designs, the proposed antenna achieves comparable bandwidth performance and larger gain per unit area with a relatively smaller size. Moreover, the simple structure renders the proposed antenna has the advantage of easy-processable and cost-effective implementation.
\end{abstract}

\section{Introduction}

Multiband band antennas are widely used in modern wireless systems, as well as radar [1]. Scholars have carried out a lot of prominent research works on the dual-band antenna. In [2], a quasi-Yagi antenna with operating bands around $1.8,2.4$, and $3.5 \mathrm{GHz}$ is proposed. The driver uniform impedance resonator (UIR) elements, arm 1 to arm 3, are excited at different frequencies as half-wave dipoles. In [3], a dual-wideband dipole directional antenna is proposed. The antenna consists of a dipole with crooked arms and parasitic $\Gamma$-shaped branches and double reflecting floors. In [4], a dual-band magnetoelectric antenna is proposed. A folded magnetic dipole antenna that is realized by a vertically assembled shorted patch is introduced to achieve wide bandwidth. In [5], a dual-band linearly polarized tag antenna for indoor positioning systems is proposed. U-shaped inverted-F antenna for the UHF band is designed to surround the dipole for the UWB band. In [6], a wideband three-dimensional dual-band magnetoelectric (ME) dipole antenna is presented for WLAN and WiMAX applications. A full-metal rectangular director is loaded right above the $\mathrm{ME}$ dipole antenna and resonates at around $3.6 \mathrm{GHz}$ due to the excitation of the long electric dipole. In [7], a dual-band $3 \times 2$ antenna arrays with $10.6 \%$ from 2.3 to $2.56 \mathrm{GHz}$ and $16.6 \%$ from 3 to $4.2 \mathrm{GHz}$ bandwidths is proposed. The antenna is realized on a FR4 substrate and a PTFE substrate. A miniaturized dual-band dipole array antenna consisting of two parallel $1 \times 4$ subarrays and three-layers of substrate for use in base stations is proposed in [8]. In [9], a $4 \times 4 \mathrm{Ku} / \mathrm{Ka}$ dual-band shared-aperture beam scanning antenna array is proposed. The concept of the structure reuse is described. To 


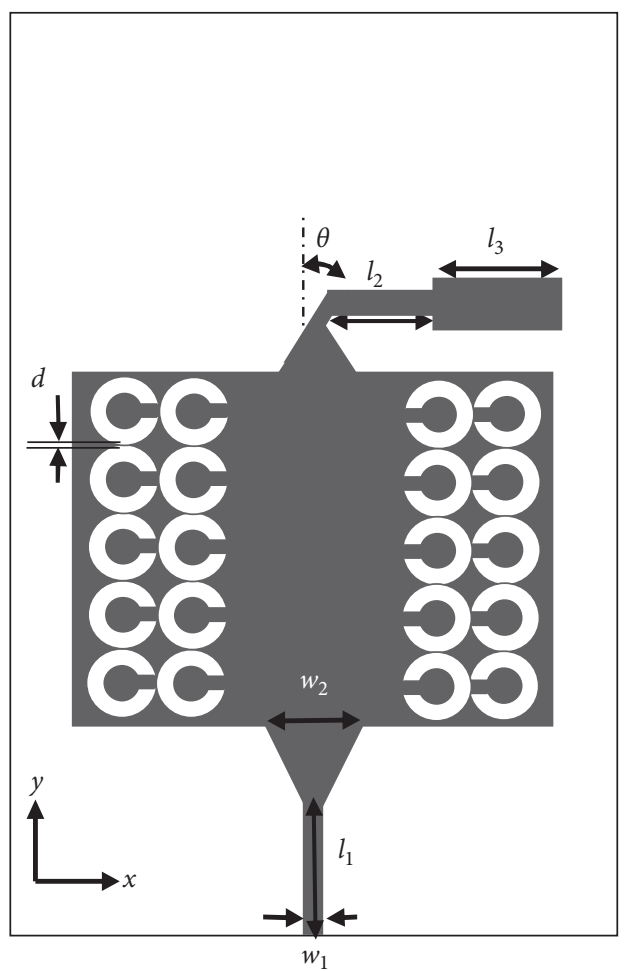

(a)

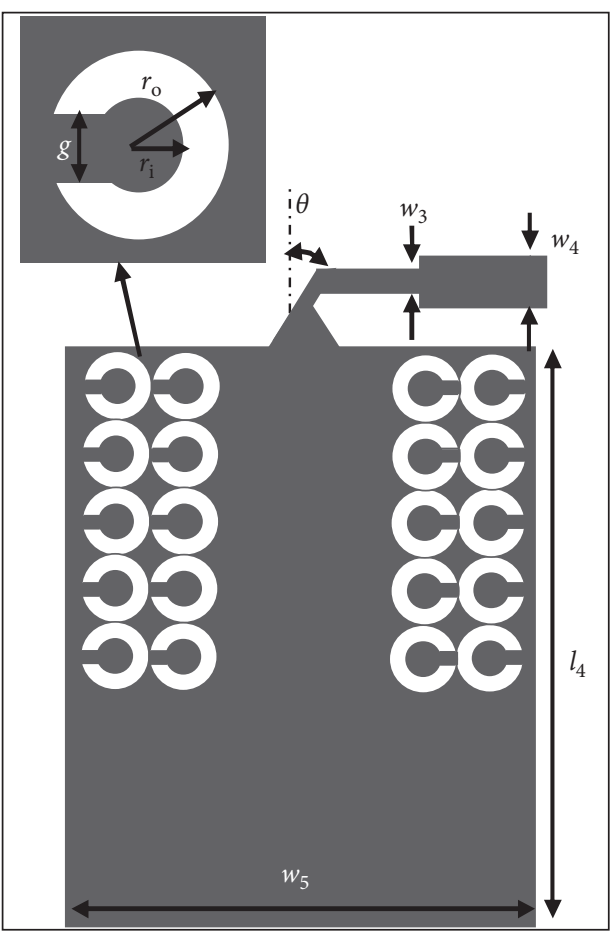

(b)

FIgURE 1: Geometry of the proposed antenna. (a) Top and (b) bottom.

TABLE 1: The structure parameters of antenna (units: $\mathrm{mm}$ ).

\begin{tabular}{lcccccccccccccc}
\hline Dimensions & $w_{1}$ & $w_{2}$ & $w_{3}$ & $w_{4}$ & $w_{5}$ & $d$ & $\theta$ & $l_{1}$ & $l_{2}$ & $l_{3}$ & $l_{4}$ & $r_{i}$ & $r_{o}$ & $g$ \\
\hline Value & 1.55 & 8 & 1.55 & 3 & 29 & 0.1 & $20^{\circ}$ & 5 & 9 & 8.7 & 34 & 1.6 & 2.4 & 0.5 \\
\hline
\end{tabular}

increase the aperture utilization efficiency, the Ku-band antenna is employed as some sidewalls of the Ka-band antenna. In [10], a metaresonator-inspired dual-band antenna for 2.4/5.2 GHz WLAN applications is proposed. Dual band operation is achieved by utilizing stacking technique on a dog-bone shaped dipole antenna. In [11], a conventional single-band printed antenna is loaded with via-less composite right/left-handed (CRLH) unit cells to achieve multiband operation. The plane of broadband transition is perpendicular to the antenna, and the structure of the whole antenna is three-dimensional. In [12], a mechanically reconfigurable dual-band slot antenna with wide tuning ranges is proposed. Although the above dual-band antennas realize good performance such as wideband or high gain, their structures are either three-dimensional or multilayer, which require a rather demanding nonplanar fabrication process and are difficult to be integrated on the planar circuits. The novel fully planar SIW based on metamaterialinspired periodic structure of coupled complementary splitring resonators (CSRR-SIW) is proposed in [13]. The CSRRSIW is an attractive candidate for a cost-effective implementation of microwave antenna due to the fully planar structure and the associated ease of implementation [14].
In our prior work [15], a broadband bow-tie antennabased CSRR-SIW is proposed. It confirms that the CSRRSIW not only confines wave propagation within the waveguide but also radiates electromagnetic waves through the CSRRs. The CSRRs produce a resonant frequency, which can be combined with the two resonant frequencies produced by the bow-tie patch and the feedline of the bow-tie patch. The bandwidth can then be broadened. Moreover, the gain in the low-frequency bandwidth is enhanced by decreasing the gap width of the CSRR.

In this article, the fully planar CSRR-SIW is applied to the design of the dual-band step impedance resonator (SIR) antenna for WLAN. First, the dual-band working mechanism is studied, including the length of the SIR element and the width of the wider strip to the antenna performance. Finally, the effect of the tilt angle of the inclined feed lines on gain enhancement of the dual-band CSRR-SIW SIR antenna is discussed. Compared with the previously reported dualband antenna designs in [7-12], the proposed antenna achieves comparable bandwidth performance and larger gain per unit area with a relatively smaller size. In addition, the proposed antenna only consists of one layer of coppercoated substrate. It can be realized by ordinary etching 


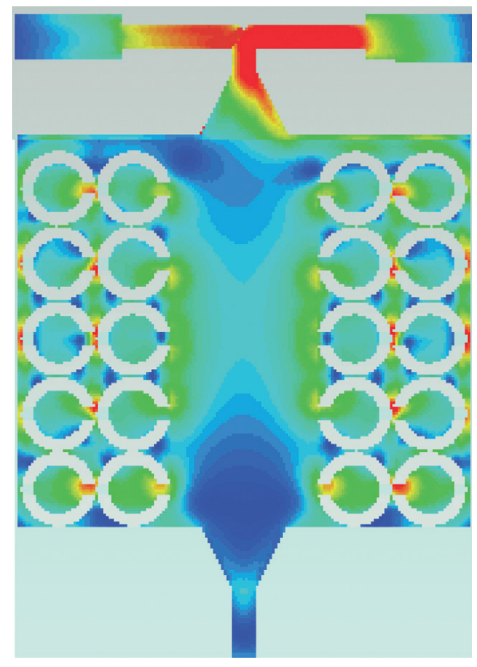

(a)
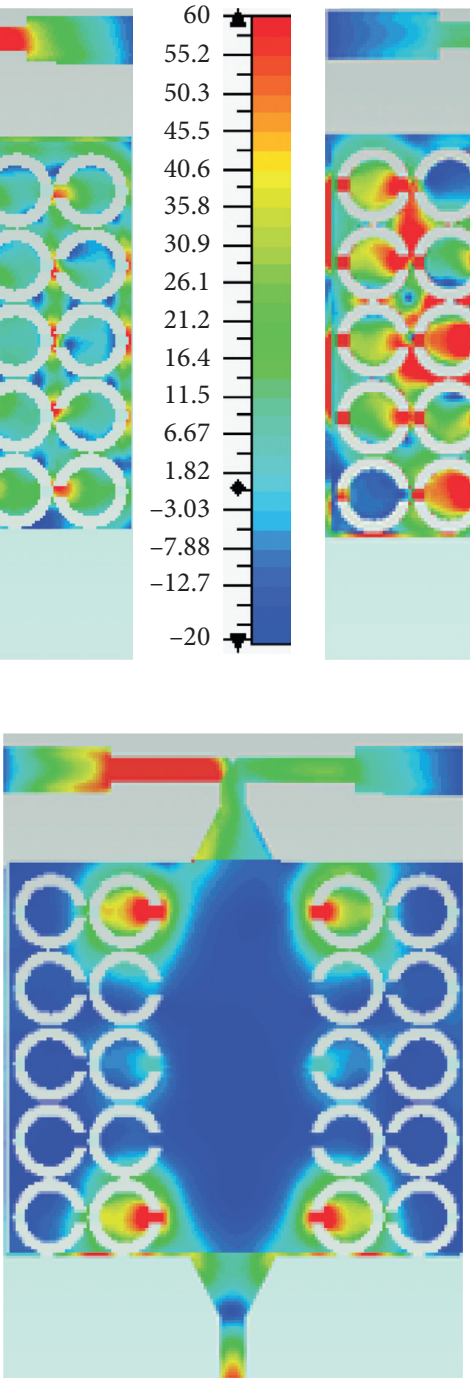

(c)
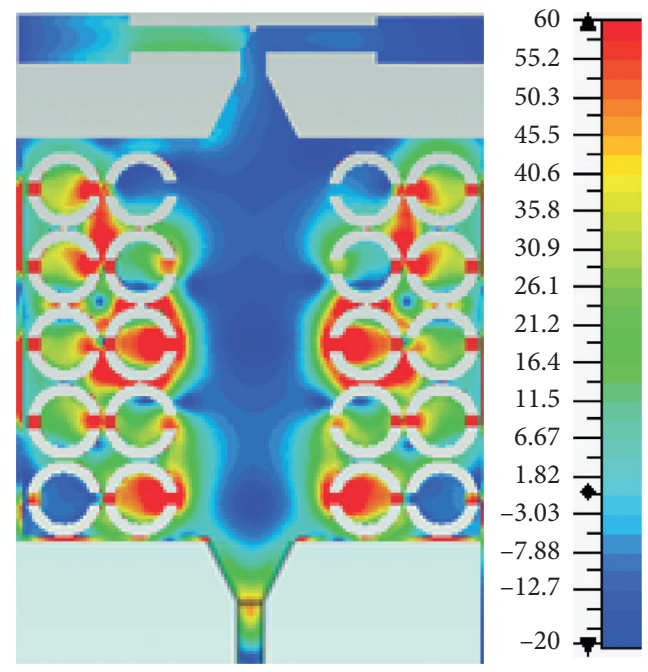

(b)

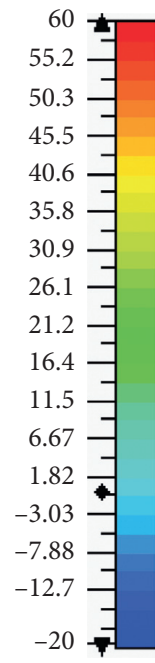

FIgURE 2: The current distribution at different frequencies over the antenna: (a) $2.4 \mathrm{GHz}$, (b) $5.2 \mathrm{GHz}$, and (c) $5.7 \mathrm{GHz}$.

techniques. Therefore, the proposed antenna structure has easy-processable and cost-effective implementation.

\section{Design of SIR Dual-Band Antenna}

2.1. Antenna Geometry. The proposed antenna is shown in Figure 1. The CSRR-SIW is the same as that in [15]. The difference is that the main element is SIR, which can achieve a dual operation bands which consists of a fundamental frequency $f_{1}$ and a second harmonic frequency $f_{\mathrm{s} 2}$. The CSRRs and two branches of the SIR element are etched on the top and bottom metal surfaces of the substrate. The substrate is Rogers 5880. The thickness of $0.504 \mathrm{~mm}$ and a dielectric constant of 2.2. Table 1 shows the dimensions of the proposed antenna.

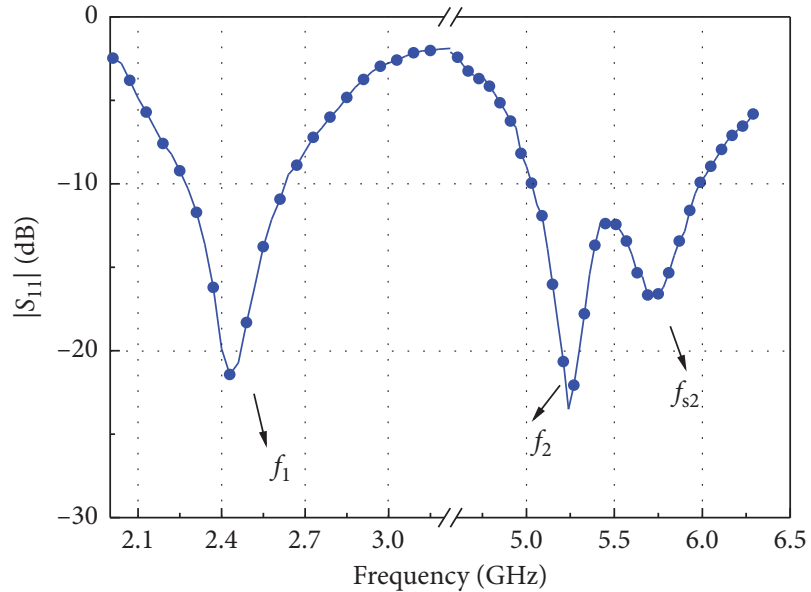

FiguRE 3: The simulated $|S 11|$ for the proposed antennas. 


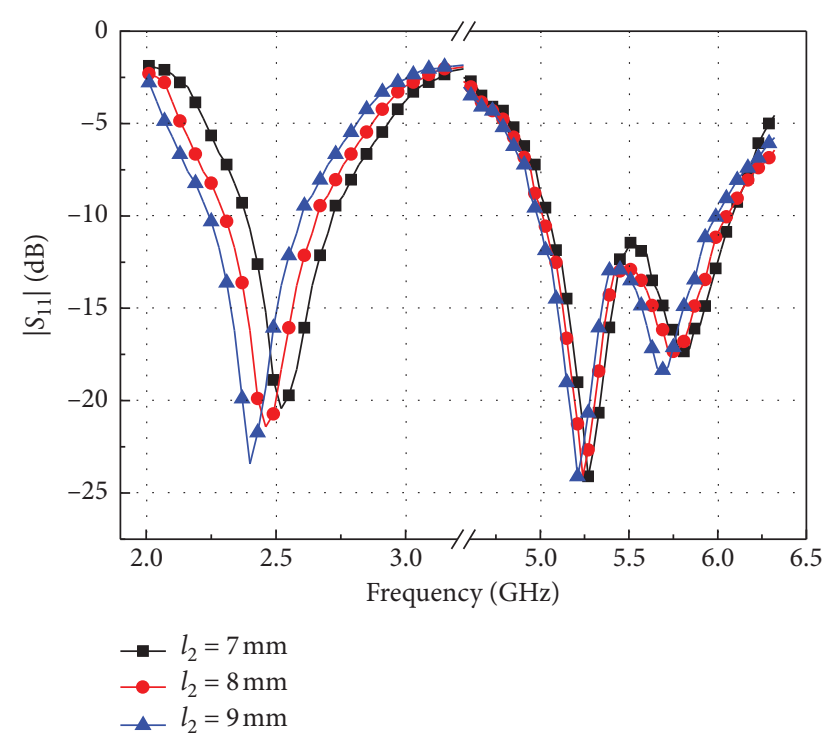

FIgURE 4: The simulated $|S 11|$ for different $l_{2}$.

2.2. Dual-Band Working Mechanism. To obtain a dual-band working, we use the SIR as the main radiating element. For the SIR, the impedance of the narrow and wider strip is $Z_{1}$ and $Z_{2}$, and the corresponding lengths is $\theta_{1}$ and $\theta_{2}$, respectively. The resonance condition can be described as follows:

$$
\tan \theta_{1} \cdot \tan \theta_{2}=R_{z}
$$

where impedance ratio $R_{z}$ is

$$
R_{z}=\frac{Z_{2}}{Z_{1}}
$$

To get a compact size, the electrical lengths $\theta_{1}$ and $\theta_{2}$ are given in [16] as

$$
\theta_{1}=\theta_{2}=\arctan \sqrt{R_{z}}
$$

On this premise, the relationship between the second harmonic frequency $f_{\mathrm{s} 2}$ and fundamental frequency $f_{1}$ is given by

$$
\frac{f_{\mathrm{s} 2}}{f_{1}}=\frac{\pi}{2 \arctan \sqrt{R_{z}}} .
$$

In this case, the appropriate resonant frequencies $f_{\mathrm{s} 2}$ and $f_{1}$ can be designed by adjusting the impedance ratio $R_{z}$. Compared with the UIR elements in [2], the SIR element has great degree of freedom in structure and design.

In this paper, we designed $f_{1}$ and $f_{\mathrm{s} 2}$ as $2.4 \mathrm{GHz}$ and $5.7 \mathrm{GHz}$, respectively. Substituting $f_{\mathrm{s} 2}$ and $f_{1}$ into (4), we obtain $R_{z}$ is about 0.6. Then, substituting $R_{z}$ into (3), we obtain $\theta_{1}=\theta_{2}=37.8^{\circ}$. Then, we get the parameters of the SIR $l_{2}, l_{3}, w_{3}$, and $w_{4}$, as shown in Table 1 .

In Figure 2, the current distribution over the antenna at 2.4 GHz, 5.2 GHz, and $5.7 \mathrm{GHz}$ are shown. We observed from Figures 2(a) and 2(c) that the current is concentrated

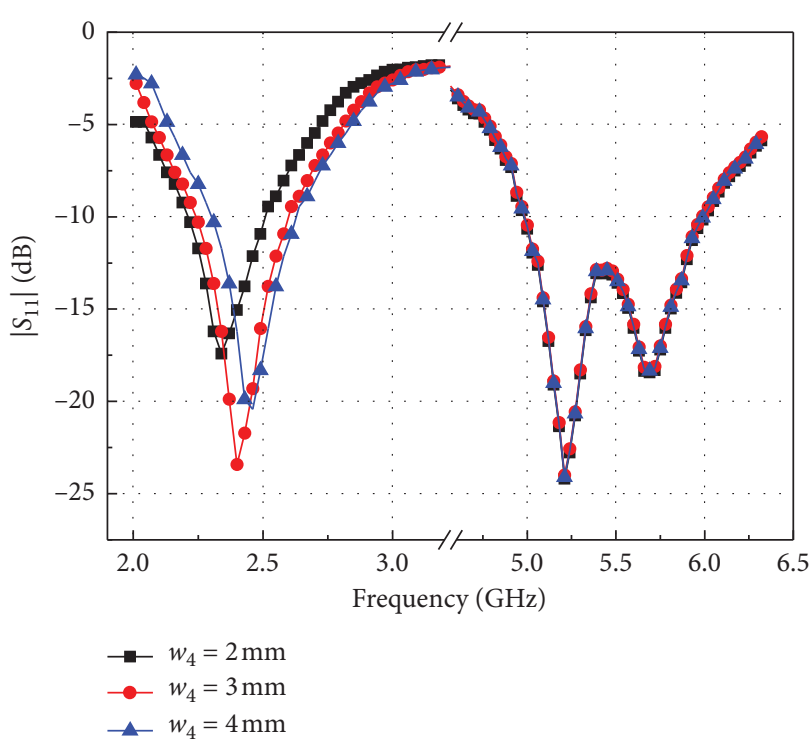

Figure 5: The simulated $|S 11|$ for different $w_{4}$.

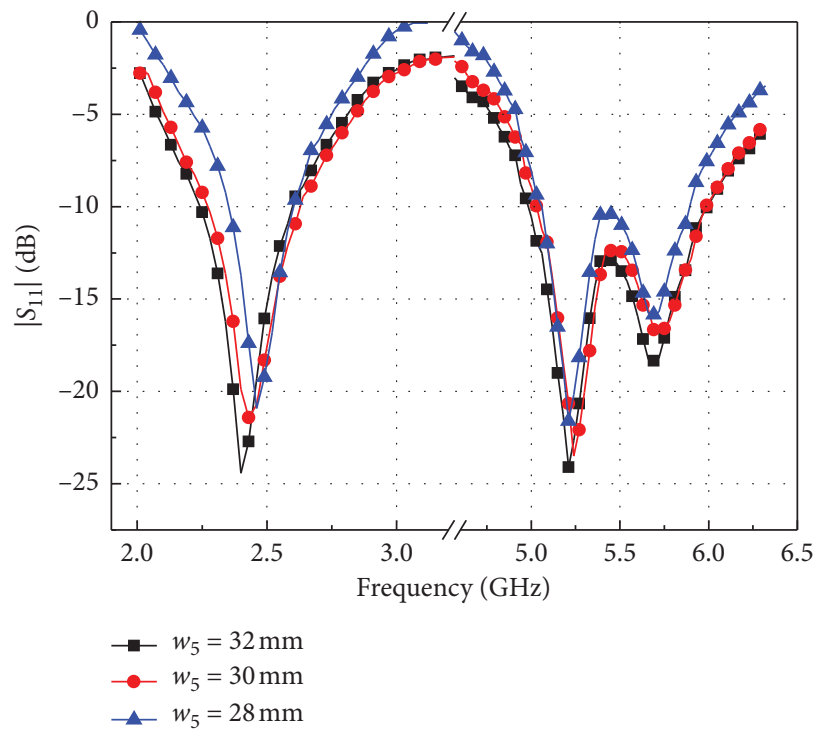

Figure 6: The simulated $|S 11|$ for different $w_{5}$.

in the SIR element at $f_{1}=2.4 \mathrm{GHz}$ and $f_{\mathrm{s} 2}=5.7 \mathrm{GHz}$. From Figure 2(b), the current is concentrated in the CSRRs at 5.2 GHz. That is to say, the SIR element produces a fundamental frequency $f_{1}$ at $2.4 \mathrm{GHz}$ and a second harmonic frequency $f_{\mathrm{s} 2}$ at $5.7 \mathrm{GHz}$. Meanwhile, the CSRRs produces a resonant frequency at high-frequency band around $5.2 \mathrm{GHz}$.

Figure 3 shows the simulated $|S 11|$ of the proposed SIR dual-band antenna. In Figure 3, there are three resonant frequencies at $f_{1}=2.4 \mathrm{GHz}, f_{2}=5.2 \mathrm{GHz}$, and $f_{\mathrm{s} 2}=5.7 \mathrm{GHz}$. The circumference of the CSRR resonant ring is approximately $\lambda / 4$ ( $\lambda$ is the wavelength at $\left.f_{2}=5.2 \mathrm{GHz}\right)$. By adjusting 


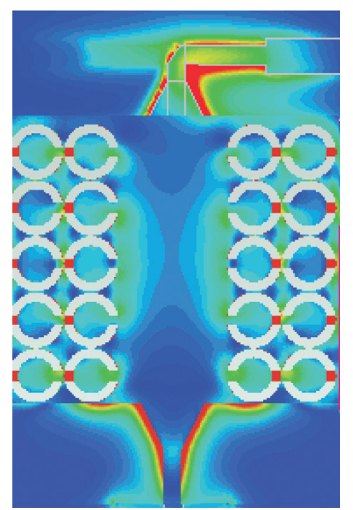

(a)

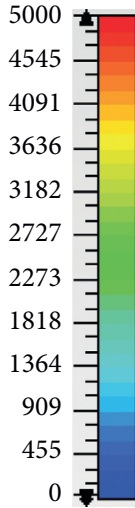

$0 \rightarrow$

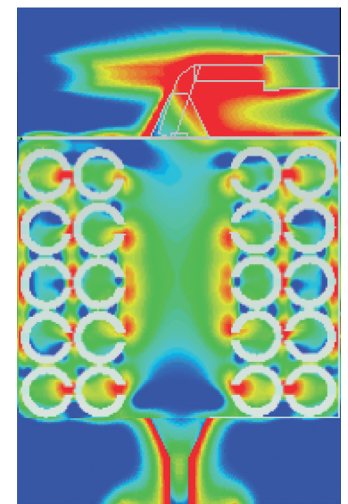

(b)

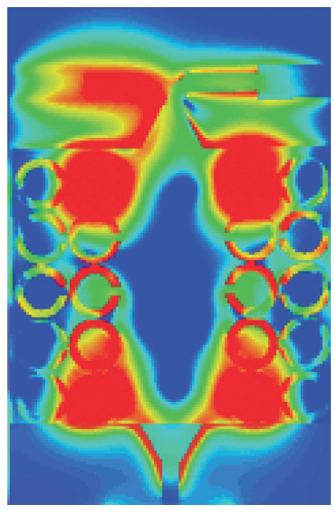

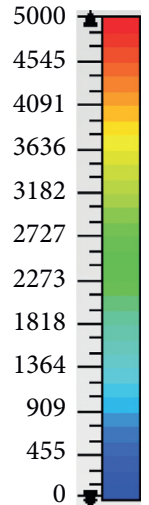

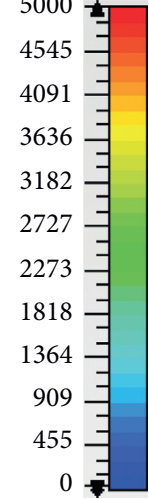

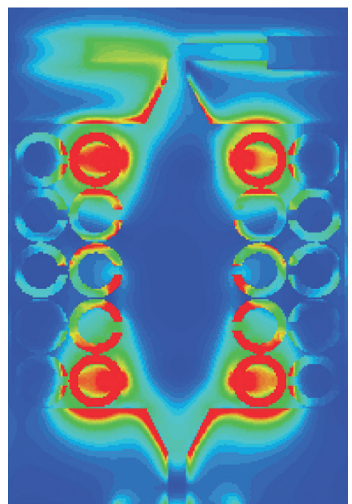

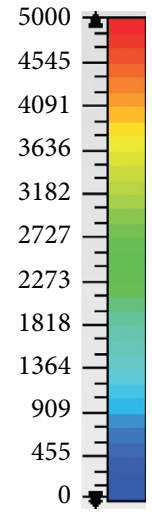

(c)

(d)

Figure 7: The E-field distribution over the antenna: (a) $\theta=0^{\circ}$ at $2.4 \mathrm{GHz}$, (b) $\theta=20^{\circ}$ at $2.4 \mathrm{GHz}$, (c) $\theta=0^{\circ}$ at $5.5 \mathrm{GHz}$, and (d) $\theta=20^{\circ}$ at $5.5 \mathrm{GHz}$.

the structure parameters, $f_{2}$ and $f_{\mathrm{s} 2}$ can be combined with each other. The high-frequency bandwidth can then be broadened. In this case, a dual-band dipole antenna based on CSRR-SIW can be obtained.

2.3. Parametric Analysis. A parametric study of key parameters based on CST MWS is given to assist with the design procedure. The results show that the length of the SIR element $l_{2}$ and $l_{3}$, the width of the wider strip $w_{4}$, and the width of the ground plane $w_{5}$ are the key parameters that affects antenna performance. Figures 4-6 show the results.

In Figure 4 , the effect of $l_{2}$ on the $|S 11|$ is shown. We observed that $l_{2}$ mainly affects the resonance at fundamental frequency $f_{1}$ and the second harmonic frequency $f_{\text {s2 }}$. With the increase of $l_{2}$, the effective electrical length $\theta_{1}$ of the narrow strip and the whole electrical length of the SIR element increases. Therefore, the fundamental frequency $f_{1}$ and the second harmonic frequency $f_{\mathrm{s} 2}$ decrease with the increasing of $l_{2}$, as shown in Figure 4. In Figure 4, when $l_{2}=7 \mathrm{~mm}$, there are three resonant frequencies: $f_{1}=2.6 \mathrm{GHz}$, $f_{2}=5.2 \mathrm{GHz}$, and $f_{\mathrm{s} 2}=5.9 \mathrm{GHz}$. The lower operation bandwidth is from $2.4 \mathrm{GHz}$ to $2.75 \mathrm{GHz}$. When $l_{2}=8 \mathrm{~mm}$, the resonance at $f_{2}$ do not change, but $f_{1}$ and $f_{\mathrm{s} 2}$ decrease to $2.47 \mathrm{GHz}$ and $5.8 \mathrm{GHz}$, respectively. The lower operation

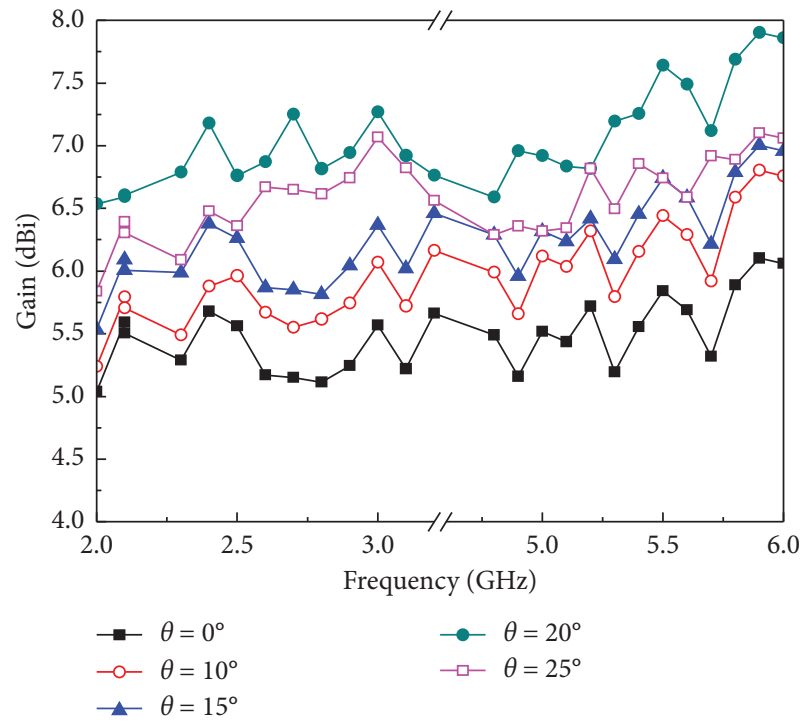

Figure 8: The simulated gain for different $\theta$.

bandwidth is from $2.3 \mathrm{GHz}$ to $2.65 \mathrm{GHz}$. When $l_{2}=9 \mathrm{~mm}, f_{1}$ and $f_{\mathrm{s} 2}$ decreases to $2.4 \mathrm{GHz}$ and $5.7 \mathrm{GHz}$, respectively. The widest lower operation bandwidth of $16.6 \%$ from $2.2 \mathrm{GHz}$ to $2.6 \mathrm{GHz}$ is obtained. The higher operation bandwidth is from $4.9 \mathrm{GHz}$ to $6 \mathrm{GHz}$. 


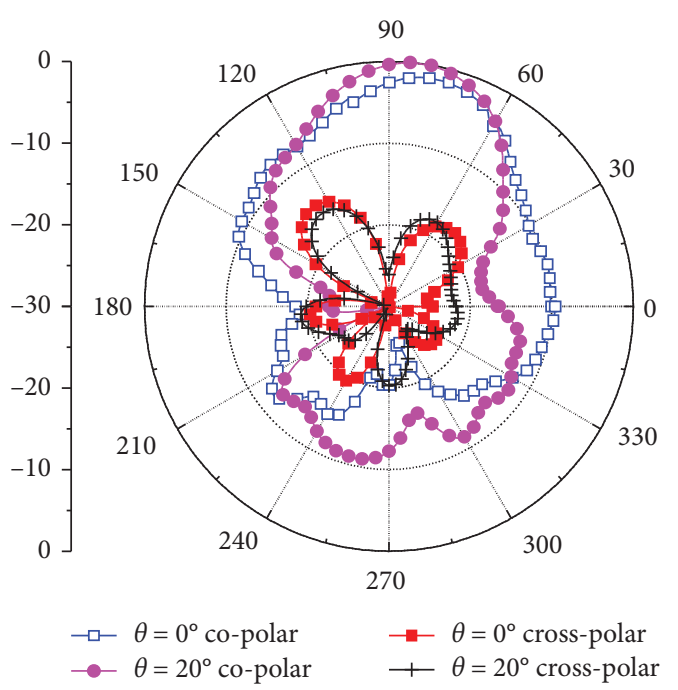

(a)

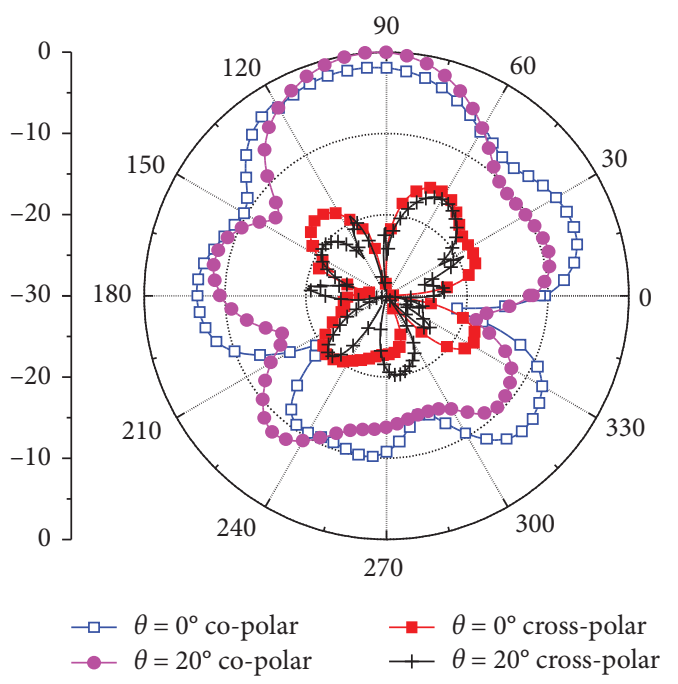

(c)

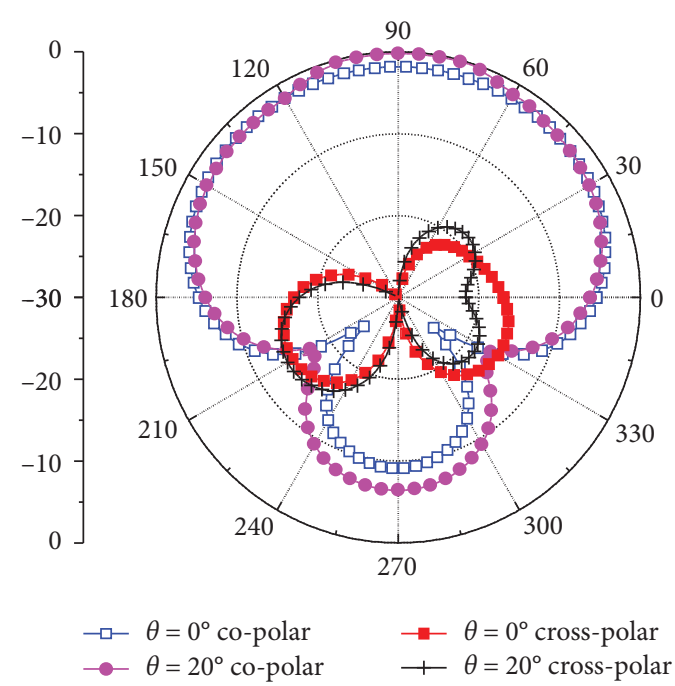

(b)

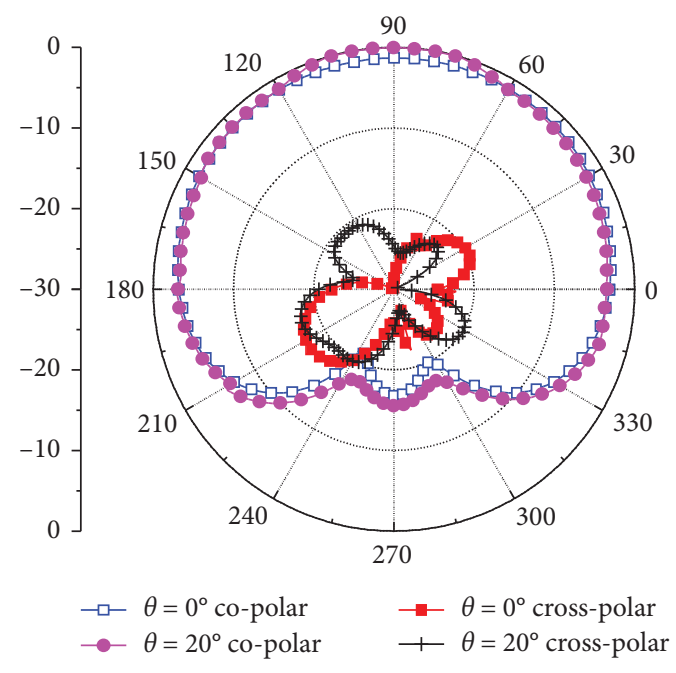

(d)

Figure 9: Simulated radiation patterns with the feed line tilted by $20^{\circ}$ and $0^{\circ}$ : (a) $2.4 \mathrm{GHz}$, E-plane, (b) $2.4 \mathrm{GHz}, \mathrm{H}-\mathrm{plane}$, (c) $5.5 \mathrm{GHz}, \mathrm{E}$ plane, and (d) $5.5 \mathrm{GHz}, \mathrm{H}$-plane.

In Figure 5, the effect of $w_{4}$ on the $|S 11|$ is shown. We observed that $w_{4}$ mainly affects the resonance at fundamental frequency $f_{1}$ near about $2.4 \mathrm{GHz}$. With the increase of $w_{4}$, the impedance ratio $R_{z}$ of the SIR element decreases. And then the whole electrical length of the SIR decreases according to (1). Therefore, the fundamental frequency $f_{1}$ increases with the increasing of $w_{4}$, as shown in Figure 5. The effective electrical length $\theta_{1}$ of the narrow strip of the SIR element has no change, and then the second harmonic frequency $f_{\mathrm{s} 2}$ almost has no change. In Figure 5, when $w_{4}=2 \mathrm{~mm}$, there are three resonant frequencies: $f_{1}=2.35 \mathrm{GHz}, f_{2}=5.2 \mathrm{GHz}$, and $f_{\mathrm{s} 2}=5.7 \mathrm{GHz}$. The lower operation bandwidth is from $2.2 \mathrm{GHz}$ to $2.5 \mathrm{GHz}$. When $w_{4}=3 \mathrm{~mm}$, the resonance at $f_{2}$ and $f_{\mathrm{s} 2}$ do not change, but $f_{1}$ increases to $2.4 \mathrm{GHz}$. The widest lower bandwidth from
$2.2 \mathrm{GHz}$ to $2.6 \mathrm{GHz}$ is obtained. When $w_{4}=4 \mathrm{~mm}, f_{1}$ increases to $2.45 \mathrm{GHz}$. And the lower operation bandwidth is from $2.3 \mathrm{GHz}$ to $2.6 \mathrm{GHz}$.

The antenna is reduced in size by reducing the width of the ground plane $w_{5}$. In Figure 6 , the effect of $w_{5}$ on the $|S 11|$ is shown. With the decrease of $w_{5}$, the electrical size of the ground plane reduced, which gives rise to a narrow bandwidth. When $w_{5}=32 \mathrm{~mm}$, the dual operation bands with bandwidths of $16.6 \%$ from $2.2 \mathrm{GHz}$ to $2.6 \mathrm{GHz}$ and $19.2 \%$ from $4.95 \mathrm{GHz}$ to $6 \mathrm{GHz}$ for $|S 11|<-10 \mathrm{~dB}$ are achieved. When $w_{5}=29 \mathrm{~mm}$, the bandwidth is $16 \%$ from $2.25 \mathrm{GHz}$ to $2.64 \mathrm{GHz}$ and $18.2 \%$ from $5 \mathrm{GHz}$ to $6 \mathrm{GHz}$, respectively. When $w_{5}=26 \mathrm{~mm}$, the bandwidths narrowed further. In addition, a further reduction of $w_{5}$ would make the width of ground plane less than the length of the SIR dipole element, 


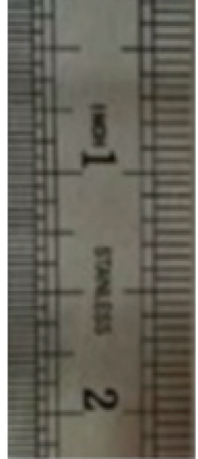

(a)

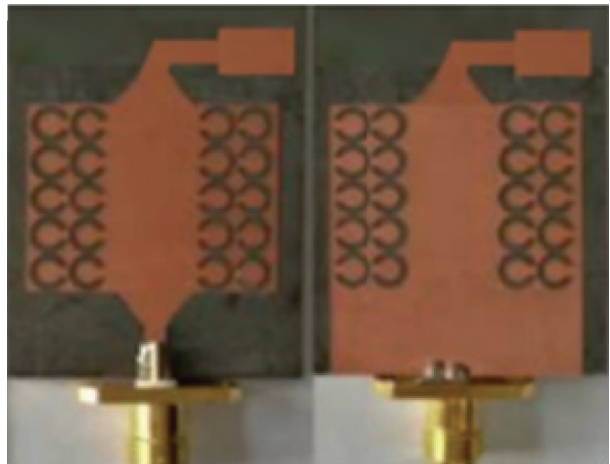

(b)

Figure 10: The photo of the antenna. (a) Top and (b) bottom.

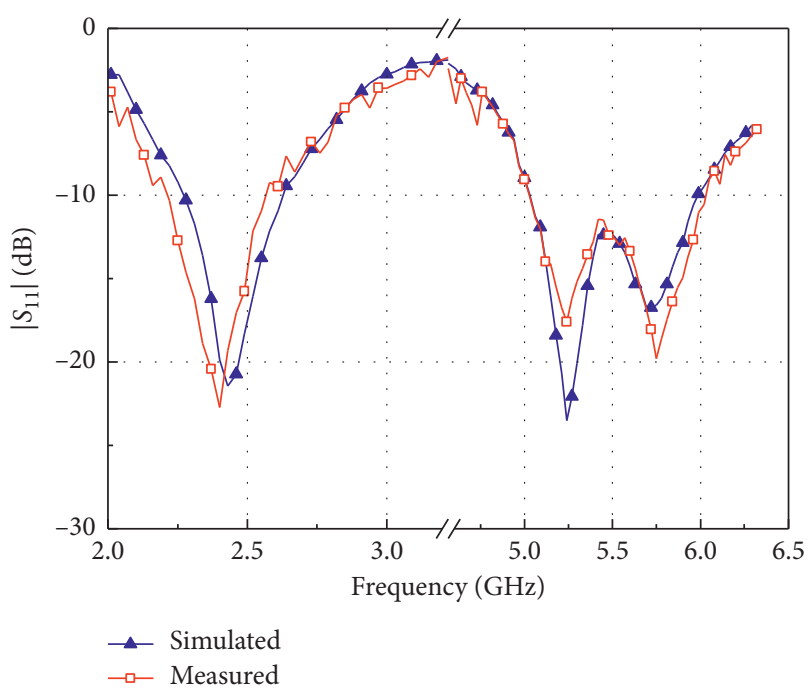

FIGURE 11: Simulated and measured S-parameters.

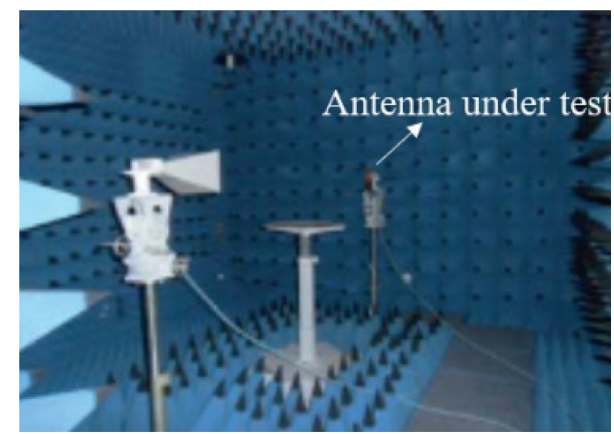

FIgURE 12: Photograph of the antenna measurement set-up.

which leads to a decrease in unidirectional radiation and a decrease in antenna gain. From the above, we choose $w_{5}=29 \mathrm{~mm}$ as the width of the proposed antenna.

\section{The Effect of Parameter $\theta$}

The two branches of the SIR element are connected by the inclined feed lines on both sides of the CSRR-SIW dual-band antenna. In this section, the effect of the tilt angle $\theta$ on gain enhancement of the dual-band CSRR-SIW SIR antenna is discussed. Figure 7 shows the E-field distribution over the antenna for different $\theta$, which clearly demonstrates that the E-field intensity increases significantly when $\theta=20^{\circ}$, whether at $2.4 \mathrm{GHz}$ or $5.5 \mathrm{GHz}$.

Figure 8 shows the effect of tilt angle $\theta$ on the peak antenna gain. With the increase of $\theta$ from $0^{\circ}$ to $20^{\circ}$, the peak gain increases, which is equivalent to enlargement of the antenna aperture [17]. A tilt angle of $\theta=20^{\circ}$ is observed to provide an optimum gain performance. Over the lower frequency band of $2.25 \mathrm{GHz}-2.64 \mathrm{GHz}$, the gain varies between $6.5 \mathrm{dBi}-7 \mathrm{dBi}$, which corresponds to a gain enhancement of $1.2 \mathrm{~dB}-1.4 \mathrm{~dB}$ compared with the antenna with $0^{\circ}$ tilt angle.

Over the higher frequency band of $5.03 \mathrm{GHz}-6 \mathrm{GHz}$, the gain varies between $7.0 \mathrm{dBi}-7.7 \mathrm{dBi}$, which corresponds to a gain enhancement of $1.7 \mathrm{~dB}-1.9 \mathrm{~dB}$ compared with the antenna with $0^{\circ}$ tilt angle.

The radiation patterns of the antenna with feed line tilted by $20^{\circ}$ and $0^{\circ}$ at $2.4 \mathrm{GHz}$ and $5.5 \mathrm{GHz}$ are shown in Figure 9 . The effective aperture increases with the parameter $\theta$, which leads to lower side-lobe level and front-to-back ratio [18]. We observed that, with the feed line tilted by $20^{\circ}$, there is a distinct improvement in the side-lobe level; however, there is degradation in the back-lobe level, especially in E-plane. From Figure 9(a), the back-lobe level is about $-20.3 \mathrm{~dB}$ and $-12.2 \mathrm{~dB}$, when $\theta=0^{\circ}$ and $20^{\circ}$ at $2.4 \mathrm{GHz}$, respectively. From Figure $9(\mathrm{c})$, the back-lobe level is about $-10.7 \mathrm{~dB}$ and $-8.2 \mathrm{~dB}$, when $\theta=0^{\circ}$ and $20^{\circ}$ at $5.5 \mathrm{GHz}$, respectively.

In addition, from Figures $7(\mathrm{a})$ and $7(\mathrm{~b})$, the E-field is mainly concentrated in the SIR element at $2.4 \mathrm{GHz}$, but there is also a part of E-field distribution around the CSRRs and the intervals among the CSRRs. Moreover, the CSRRs are etched side-by-side on the top and bottom metal surfaces of the substrate, with the complementary gaps facing toward opposite directions. Therefore, there are certain phase difference in the E-field distribution around here. Therefore, even at $2.4 \mathrm{GHz}$, the maximum radiation patterns of E-plane have a deviation of less than 8, shown in Figure 9(a). From Figures 7 (c) and 7(d), the CSRRs are similar to a slot array, and each CSRR has certain phase difference at $5.5 \mathrm{GHz}$. Therefore, the maximum radiation patterns of E-plane deviate a certain angle, as shown in Figure 9(c). 


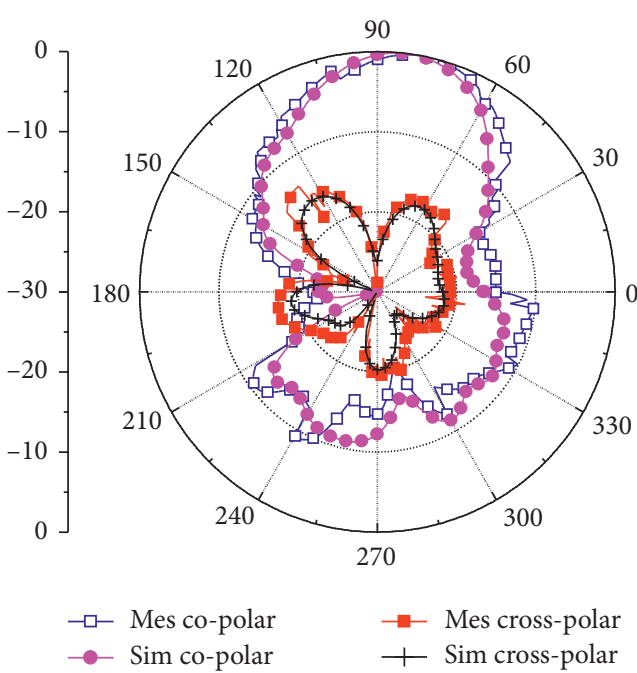

(a)

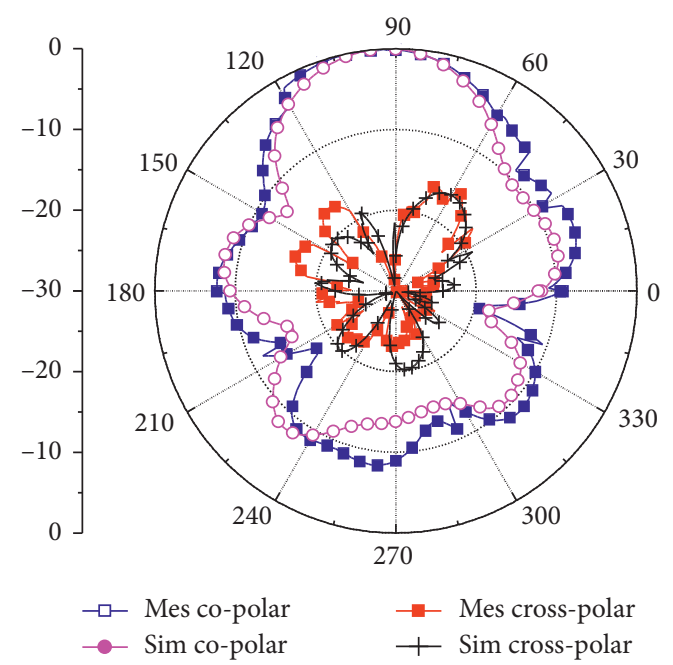

(c)

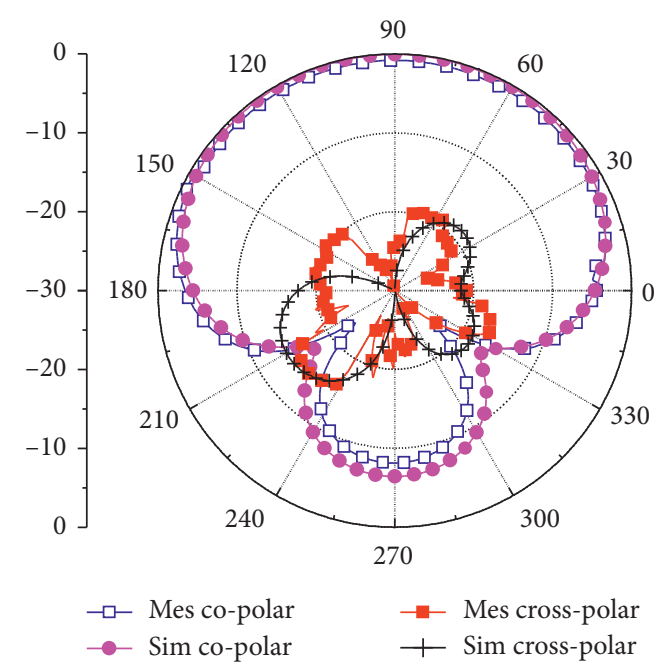

(b)

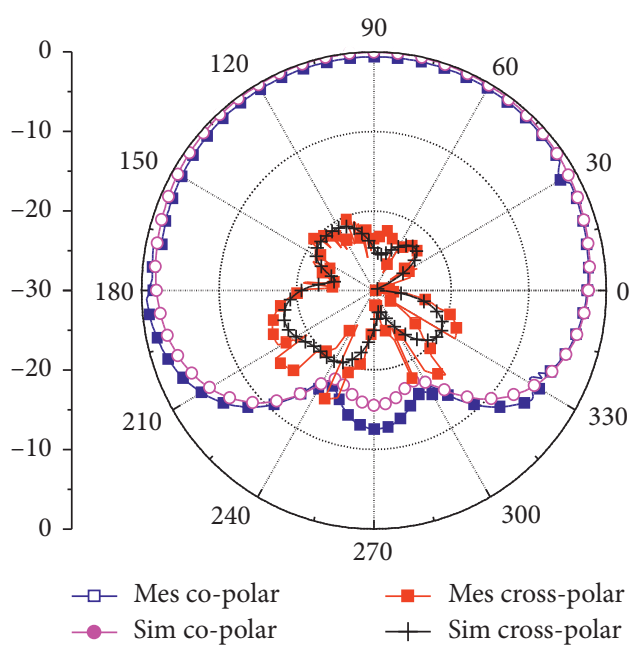

(d)

FIGURE 13: Simulated and measured radiation patterns: (a) $2.4 \mathrm{GHz}$, E-plane, (b) $2.4 \mathrm{GHz}, \mathrm{H}$-plane, (c) $5.5 \mathrm{GHz}$, E-plane, and (d) $5.5 \mathrm{GHz}$, H-plane.

\section{The Measured Results and Discussion}

The realized antenna is shown in Figure 10. The network analyzer Agilent N5230C is used to measure the magnitude of $|S 11|$. The simulated and measured $|S 11|$ are shown in Figure 11. We observed that the lower operation band is $16 \%$ from $2.25 \mathrm{GHz}$ to $2.64 \mathrm{GHz}$, and the higher operation band is $18.2 \%$ from $5 \mathrm{GHz}$ to $6 \mathrm{GHz}$. The measured result agrees well with the simulated one.

Figure 12 shows the anechoic chamber and the testing system for antenna radiation pattern. Figure 13 shows the normalized radiation patterns. The measured result agrees well with the simulated one. Figure 14 shows the measured gain and radiation efficiency of the dual-band CSRR-SIW antenna. We observed that the gain within the operating frequency range is flat. Maximum gain of the antenna is found to be $7 \mathrm{dBi}$ for the lower operation band and $7.7 \mathrm{dBi}$ for higher operation band. Also, the radiation efficiency is higher than $82 \%$ for the dual operation bands.

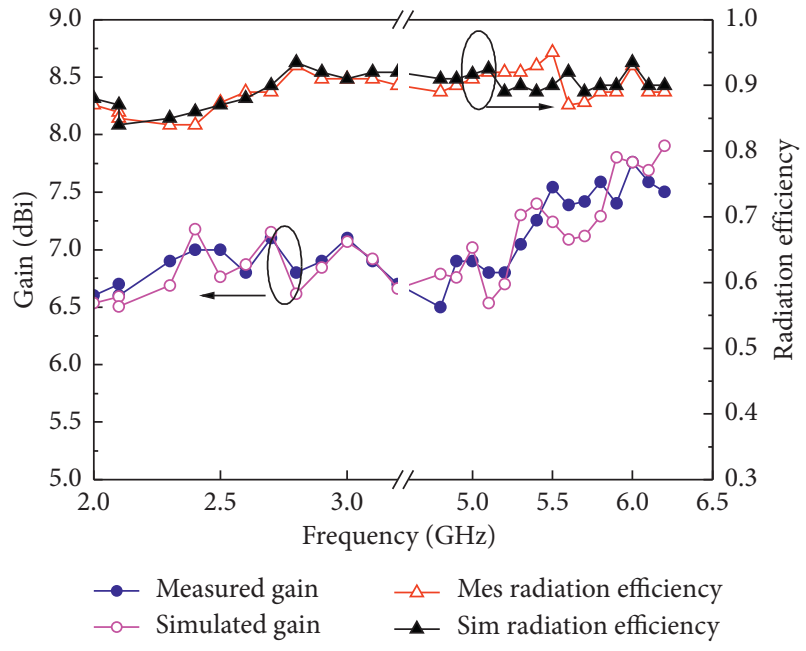

FIGURE 14: Measured and simulated gain and radiation efficiency for the proposed antenna. 
TABLE 2: Comparison to reported dual-band or multifrequency antenna and arrays.

\begin{tabular}{lcccc}
\hline Ref. & Bandwidth (low-/high-band) & Elements no. & Dimension $\left(\lambda_{0}\right)$ & Gain $($ low-/high-band) $(\mathrm{dBi})$ \\
\hline$[2]$ & $7.8 \% / 30.1 \% / 12.5 \%$ & Single & $0.35 \times 0.35$ & $2.3 / 2.8 / 4.1$ \\
{$[7]$} & $10.6 \% / 16.6 \%$ & $3 \times 2$ & $1.1 \times 1.83$ & $14.3 / 16.2$ \\
{$[8]$} & $14.9 \% / 13.2 \%$ & $2 \times 4$ & $3.35 \times 0.98$ & $12.1 / 12.4$ \\
{$[9]$} & $9.3 \% / 4.3 \%$ & $4 \times 4$ & $2.83 \times 1.73$ & $13.2 / 17.2$ \\
{$[10]$} & $12 \% / 10.5 \%$ & Single & $0.3 \times 0.33$ & $4.5 / 7$ \\
{$[11]$} & $4.18 \% / 12.2 \%$ & Single & $0.53 \times 0.41$ & $0.5 / 2.3$ \\
{$[12]$} & $17 \% / 8.25 \%$ & Single & n.a. & $4.5 / 6.8$ \\
This work & $16 \% / 18.2 \%$ & Single & $0.23 \times 0.34$ & $7 / 7.7$ \\
\hline
\end{tabular}

The performances of the proposed dual-band SIR antenna with the previously reported dual-band or multifrequency antennas are compared, and the results are listed in Table 2. The proposed antenna achieves comparable bandwidth performance and larger gain per unit area with a relatively smaller size. Moreover, the simple structure renders that the proposed antenna has the advantage of easyprocessable and cost-effective implementation.

\section{Conclusion}

A dual-band SIR antenna based on metamaterial-inspired CSRR-SIW was proposed. The SIR element produces a fundamental frequency $f_{1}$ at $2.4 \mathrm{GHz}$ and a second harmonic frequency $f_{\mathrm{s} 2}$ at $5.7 \mathrm{GHz}$. Meanwhile, the CSRRs produces a resonant frequency at high-frequency band around $5.2 \mathrm{GHz}$, which can be combined with the second harmonic frequency $f_{\mathrm{s} 2}$. The fabricated antenna provided a dual operation bands with bandwidths of $16 \%$ from $2.25 \mathrm{GHz}$ to $2.64 \mathrm{GHz}$ and $18.2 \%$ from $5 \mathrm{GHz}$ to $6 \mathrm{GHz}$ for $|S 11|<-10 \mathrm{~dB}$. The maximum gains of $7 \mathrm{dBi}$ and $7.7 \mathrm{dBi}$ for the lower and higher operation bands were obtained, respectively. The proposed antenna achieved comparable bandwidth performance and larger gain per unit area with a relatively smaller size. In addition, the proposed antenna has the advantage of costeffective implementation thanks to the simple structure.

\section{Data Availability}

The data used to support the findings of this study are available from the corresponding author upon request.

\section{Conflicts of Interest}

The authors declare that they have no conflicts of interest.

\section{Acknowledgments}

This work was supported by the Youth Science and Technology Research Foundation of Shanxi Province, China (201901D211432), Science and Technology Innovation Group of Shanxi Province, China (201805D131006), Science and Technology Innovation Program of Institutions of Higher Education of Shanxi Province, China (2020L0466), Key Research and Development Projects (Industry) of Datong, China (2019030), and General Science Projects of Shanxi Datong University (2019K1).

\section{References}

[1] N. Haider, D. Caratelli, and A. G. Yarovoy, "Recent developments in reconfigurable and multiband antenna technology," International Journal of Antennas and Propagation, vol. 2013, Article ID 869170, 14 pages, 2013.

[2] Y. Ding, Y. C. Jiao, P. Fei, B. Li, and Q. T. Zhang, "Design of a multiband quasi-yagi-type antenna with CPW-to-CPS transition," IEEE Antennas and Wireless Propagation Letters, vol. 10, pp. 1120-1123, 2011.

[3] H. Zhang, Y.-C. Jiao, and Z. Weng, "A novel dual-wideband directional dipole antenna with double reflecting floors," IEEE Antennas and Wireless Propagation Letters, vol. 16, pp. 1941-1944, 2017.

[4] K. He, S.-X. Gong, and F. Gao, "A wideband dual-band magneto-electric dipole antenna with improved feeding structure," IEEE Antennas and Wireless Propagation Letters, vol. 13, pp. 1729-1732, 2014.

[5] W. An, Z. Shen, and J. Wang, "Compact low-profile dual-band tag antenna for indoor positioning systems," IEEE Antennas and Wireless Propagation Letters, vol. 16, pp. 400-403, 2017.

[6] J. Tao, Q. Feng, and T. Liu, "Dual-wideband magnetoelectric dipole antenna with director loaded," IEEE Antennas and Wireless Propagation Letters, vol. 17, no. 10, pp. 1885-1889, 2018.

[7] Z. Wang, G.-x. Zhang, Y. Yin, and J. Wu, "Design of a dualband high-gain antenna array for WLAN and WiMAX base station," IEEE Antennas and Wireless Propagation Letters, vol. 13, pp. 1721-1724, 2014.

[8] M. Li, Q. Li, B. Wang, C. Zhou, and S. Cheung, "A miniaturized dual-band base station array antenna using band notch dipole antenna elements and AMC reflectors," IEEE Transactions on Antennas and Propagation, vol. 66, no. 6, pp. 3189-3194, 2018.

[9] Y. R. Ding and Y. J. Cheng, "Ku/ka dual-band dual-polarized shared-aperture beam scanning antenna array with high isolation," IEEE Transactions on Antennas and Propagation, vol. 67, no. 4, pp. 2337-2342, 2019.

[10] S. V. Pushpakaran, R. K. Raj, P. V. Vinesh, R. Dinesh, P. Mohanan, and K. Vasudevan, "A metaresonator inspired dual band Antenna for wireless applications," IEEE Transactions on Antennas and Propagation, vol. 62, no. 4, pp. 2287-2291, 2014.

[11] K. Saurav, D. Sarkar, and K. V. Srivastava, "CRLH unit-cell loaded multiband printed dipole antenna," IEEE Antennas and Wireless Propagation Letters, vol. 13, pp. 852-855, 2014.

[12] I. T. Nassar, H. Tsang, D. Bardroff, C. P. Lusk, and T. M. Weller, "Mechanically reconfigurable, dual-band slot dipole antennas," IEEE Transactions on Antennas and Propagation, vol. 63, no. 7, pp. 3267-3271, 2015.

[13] M. Nitas, M.-T. Passia, and T. V. Yioultsis, "Analysis and design of a CSRR-based fully planar substrate-integrated 
waveguide for millimeter-wave circuits and antennas," in Proceedings of the 2017 11th European Conference on Antennas and Propagation (EUCAP), pp. 19-24, Paris, France, March 2017.

[14] M.-T. Passia, M. Nitas, and T. V. Yioultsis, "A fully planar antenna for millimeter-wave and $5 \mathrm{G}$ communications based on a new CSRR-enhanced substrate-integrated waveguide," in Proceedings of the 2017 International Workshop on Antenna Technology: Small Antennas, Innovative Structures, and Applications (iWAT), pp. 1-3, Athens, Greece, March 2017.

[15] C. Feng, T. Shi, and L. Wang, "Novel broadband bow-tie antenna based on complementary split-ring resonators enhanced substrate-integrated waveguide," IEEE Access, vol. 7, pp. 12397-12404, 2019.

[16] M. Makimoto and S. Yamashita, Microwave Resonators and Filter for Wireless Communication, pp. 12-96, Springer, Berlin, Germany, 2001.

[17] A. Dadgarpour, B. Zarghooni, B. S. Virdee, and T. A. Denidni, "Millimeter-wave high-gain SIW end-fire bow-tie antenna," IEEE Transactions on Antennas and Propagation, vol. 63, no. 5, pp. 2337-2342, 2015.

[18] G. Zhai, Y. Cheng, Q. Yin, L. Chiu, S. Zhu, and J. Gao, "Super high gain substrate integrated clamped-mode printed logperiodic dipole array antenna," IEEE Transactions on Antennas and Propagation, vol. 61, no. 6, pp. 3009-3016, 2013. 